\title{
ROMANISCHE RELIKTE IM DREILÄNDERECK A-CH-I
}

Wer aus einem Gebiet stammt, in dem sich mehrere Sprachen nach einem längeren Ablösungsprozeß überlagert haben, der grübelt unwillkürlich manchem Wort und mancher Wendung nach, die man aus dem heutigen Sprachgebrauch nicht erklären kann. So findet man im heute deutschsprachigen Montafón (Vorarlberg), im Paznáun und Stanzertal (Verwall- und Arlberggebiet), im sog. Oberen Gericht (oberstes Tiroler Inntal) - Nauders - und im oberen Vintschgau (oberstes Etschtal in Südtirol, Italien) noch in unserer Zeit manche Gemeinsamkeiten mit dem angrenzenden Samnáun (Schweiz) und dem rätoromanischen Unterengadin (unteres Schweizer Inntal). Wie eigenwillig die sprachliche Realität, an der bekanntlich viele Faktoren mitwirken, schließlich ausgeprägt sein kann, zeigen uns schon die jeweiligen Talschaftsnamen.

Das Montafón mda. [munt $\alpha$ fû] hat im heimischen niederalemannischen Dialekt Endsilbenbetonung wie auch Paznáun ${ }^{1}$, beide mit - ONE gebildet wie übrigens auch tir. Samnáun (< St. MAGNUS), rtr. Samagnún. Das ist bei mehrsilbigen Namenformen schon ein wichtiger Hinweis auf vordeutsche Herkunft und Übernahme bis gegen 1200 , obwohl auch deutsch gebildete Namenformen - durch Überentäußerung - im Süddeutschen nicht selten Endbetonung aufweisen wie Brätblies, Faralóch ${ }^{2}$ etc. Der Name Nauders gegenüber rtr. Danuder gibt wenig Neues her, denn die Diphthongierung von $\hat{u}>a u$ im 12. Jht. (wie auch $\hat{\imath}>a ́ i, \ddot{u}>\ddot{a} u$ ) durch das Bairische ${ }^{3}$ kennen wir schon aus Paznaun und Samnaun, es sei denn die im Rätoromanischen nicht selten agglutinierte Präposition, die auch Damal, Dumaist, Dür etc. (dt. Mals, Imst, Eyrs) zeigen ${ }^{4}$. Diese Eigenheit des Romanischen

1 Montafón ist wohl MONS 'Alpe' + TUBUS 'Tobel, Rinne', vgl. J. Zehrer im Jahrbuch d. Vbger. Landesmus. Vereins 104 (1960) 134. Paznáun zu PUTEUS + INU, das als Ortsname Pazín und als Reliktwort Putzen ergab, vgl. K. Finsterwalder in Jahrbuch d. Österr. Alpenvereins 80 (1955) 35. Bei O. Stolz, Politisch-historische Landesbeschreibung von Tirol I/2 im Archiv f. Österr. Geschichte 107/2 (1925) 772 ff. "in Patzenun" ohne Diphthong; Belege für Galtür, dessen Walser seit 1320 nach Nauders zinsten, da sie zum Unterengadin gehörten.

2 Vgl. W. Vogt, Vorarlberger Flurnamenbuch I/2, Montafon, Bregenz 1973, Gaschurn Nr. 109, 176: Dt. Bréitblies. Fárnloch.

3 Deutsche Lautverschiebung, Umlaut und Diphthongierung werden seit W. Bruckner für eine relative Chronologie herangezogen, insbes. von K. Finsterwalder in ZONF 4 (1929) 228 ff. und in Tiroler Heimat 26 (1962) $82 \mathrm{ff}$. etc., ebenso von H. Kuen in Ladinia 9 (1985) 19-29.

4 Vgl. K. von Ettmayer, Geographia raetica, in: GRM 2 (1910) 302.

Wir zitieren in der Folge abgekürzt im Text:

DRG = Dicziunari rumantsch grischun, bearbeitet von R. von Planta, A. Schorta u.a., Chur 1939 ff., bisher 7 Bde.

FISCHER, H. - W. Pfleiderer, Schwäbisches Wörterbuch, Tübingen 1904-36, 6 Bde.

IDIOTIKON = Schweizerisches Idiotikon, Wörterbuch der schweizerdeutschen Sprache, Frauenfeld $1881 \mathrm{ff}$. 
muß gestützt worden sein durch den unterschiedslosen Gebrauch etwa des AD bei der romanischen Orts- wie auch der Zielangabe (dt. im/in dem vs. in den Wald) gegenüber der Unterscheidung im Deutschen: wo? vs. wohin? mit entsprechend unterschiedlichen Folgekonstrukten ${ }^{5}$.

Der Vintschgau, früh belegt als Venustica Vallis ${ }^{6}$, hat im ital. Val Venósta die alte Betonung auf der Ableitungssilbe, die durch eine im Lateinischen auffällige Lautfolge (wie einst in Trieste, Este, Imst) die Bewohner schon in römischer Zeit als vorrömisch kennzeichnete: Venóstes führt geradlinig zu rtr. Vnuost, allerdings mit der radikalen Kürzung der unbetonten Silben, die schließlich auch - aber erstsilbenbetont - bei bair. Vinsch(t)gau endet. Dt. Engadin ist dagegen eine Mischform, ein Kompromiß zwischen altem vallis Eniatína a. 930 und bair. Engedein ${ }^{7}$, heute oengad. Engiadigna, surm. Naschadoina, surs. Giadina, im 13. Jht. auch Engdina a.ä.; der Name hängt mit Aenus bzw. Inn zusammen und wurde als Ableitung von *Eniates 'Inn-Anwohner' (R. von Planta) oder direkt vom keltischen Flußnamen (J.U. Hubschmied) erklärt. Von Grenzen an Wasserscheiden spürt man hier wenig, denn das rätoromanische Münstertal/Müstáir ( $<$ MONASTERIUM) ${ }^{8}$ liegt jenseits des Ofenpasses, das vorrömische Nauders (am Stillebach, nicht an der Etsch) gehört sprachlich anscheinend eher zum Vintschgau, das Samnaun ist halb schweizerisch, halb deutsch und war bis vor einigen Jahrzehnten noch mehrsprachig. Galtür $<$ CULTURA im obersten Paznaun, auch als Galtyr und Cultaur belegt, auch Galtura a. 1624 (Stolz 1925, 774) hat noch heute eigenwillige (hochalemannische) Walserspuren wie auch das angrenzende innere Montafon ${ }^{9}$.

JUTZ, L., Vorarlbergisches Wörterbuch mit Einschluß des Fürstentums Liechtenstein, Wien 1960-65, 2 Bde.

KLUGE, F., Etymologisches Wörterbuch der deutschen Sprache, bearbeitet von E. SEEBOLD, Berlin 221989.

LUTTA, C.M., Der Dialekt von Bergün, Halle 1923.

PALLIOPPI, Z. und E., Dizionari dels idioms romantschs d'Engiadin' ota e bassa, Samedan 1895. PEER, O., Dicziunari rumantsch ladin-tudais-ch, Chur 1962.

REW $=$ Meyer-Lübke, W., Romanisches etymologisches Wörterbuch, Heidelberg ${ }^{5} 1972$.

RN = Rätisches Namenbuch, hg. von R. von Planta, A. Schorta und K. Huber, Zürich-Leipzig 1939-86, 3 Bde.

SCHATZ, J., Wörterbuch der Tiroler Mundarten, hg. von K. Finsterwalder, Innsbruck 1955-56. SCHÖPF, J.B., Tirolisches Idiotikon, Innsbruck 1866.

5 Vgl. dazu H. Lüdtke, Präpositionen der Orts-, Höhen- und Richtungsbezeichnung im Graubündner Oberland, in: RF 66 (1955) 374-378 und Th. Ebneter, Schu' I Schvob 'ins Schwabenland', in: Fakten und Theorien, Fs. für H. Stimm, Tübingen 1982, 59-70 sowie ders., Die Adverbien und Präpositionen des Ortes und der Richtung im Romanischen von Vaz/Obervaz, in: ZrP 100 (1984) 387-407.

6 Im 8. Jht. bei Arbeo von Freising erstmals genannt in dieser Form, vgl. O. Stolz, Die Ausbreitung des Deutschtums in Südtirol im Lichte der Urkunden Bd. IV, 19 f., im 16. Jht. bei Chiampell Vnuest; im lombardischen Veltin schon im 13. Jht. Venosta, ebenso 1283 bei Vintschgauer Notaren; seit 1077 auch in der verdeutschten Form Finsgowe, sogar in lateinischen Urkunden. Vgl. K. Finsterwalder, Geschichte der Namen - Geschichte der Sprachen im Obervintschgau, in: Jahrbuch des Südtiroler Kulturinstitutes 5-7 (1965-67) 222-245.

7 Ältere Belege K. von Ettmayer 1910, 305 und jetzt übersichtlich im RN 2, 680 f., Th. Gartner schreibt in seiner Rtr. Gram. 1883, seltener im Handbuch 1910 noch Engedein.

8 Nach RN 2, $764 \mathrm{f}$. älter Monasterium Tuberis a. 881, M. quod Duberis dicitur a. 888, dann Tubris a. 1087 und nicht Z-.

9 Vgl. R. Jaufer, Die romanischen Orts- und Flurnamen des Paznauntales, Innsbruck 1970, 58 und vor allem das noch ungedruckte Jahrzeitbuch von Bartholomäberg (15. Jht.). 
In dem ganzen, relativ hoch gelegenen und nach drei verschiedenen Meeren (zu Rhein, Donau, Adria) hin entwässerten Dreiländereck gibt es eine beachtliche Zahl verbindender Gemeinsamkeiten, die frühere politische Bindungen oder der Reiseund Warenverkehr allein nicht zu erklären vermögen. Während im östlichen Bairischen heute das häufigere Wort hören zu sein scheint (Schatz 301; vgl. Interjektion hearst etc.), das in Tirol und bes. in Vorarlberg meist als 'beenden, aufhören' verstanden wird (Jutz I, 1443) und in dieser Bedeutung auch zlad. scuté semantisch abgelenkt hat, verwendet unser Dreiländereck losen, und zwar großteils mit Nasaleinschub, den ich sonst nur in Randgebieten wie Osttirol oder in Sprachinseln ${ }^{10}$ als Archaismus belegen kann:

losn $\alpha$ nach Jutz 2, $300 \mathrm{f}$. in Bludenz, Brand, Montafon;

lousne $(n)$ nach Schatz 396 im Oberinntal11, aber nicht im Vintschgau, wie mir Heimische sagen. Nach dem Idiotikon 3, 1446 ist diese Variante in der Schweiz nicht zu belegen.

Das Verb losen 'horchen, lauschen, zuhören' ist natürlich in einem weit gröBeren Gebiet vertreten, wie auch der Flurname $L u s a^{12}$ zeigt, aber die "doppelte" Infinitivbildung ${ }^{13}$ hat regional engeren Indizienwert und spricht zumindest für $\mathrm{Zu}$ sammenhang, ähnliche Grundlagen und gleichartige Entwicklung in neuerer Zeit. Aus diesem Verband fällt am ehesten der Vintschgau, der nur langsam und relativ spät, wohl von Meran und Schlanders her, seine neue südbairische Umgangssprache übernommen hat ${ }^{14}$.

Wenn wir auf die nächste Sprachschichte zurückgehen, nämlich auf das Rätoromanische, so stellt dieses im Engadin noch immer die Haus- und Umgangssprache dar, neben der aber fast alle Heimischen auch das Deutsche (zumindest Schweizerdeutsch) und die meisten auch Italienisch beherrschen. In meiner Heimatmundart (Bürs/Bludenz, südlicher Walgau in Vorarlberg) gibt es nun eine ganze Reihe von

10 Vgl. lisnen (kärtn. Grenze) Schöpf 393, nach eigenen Aufnahmen auch im Paznaun ab See: W. Tschinkel, Wörterbuch der Gottscheer Mundart, Wien 1973/76, Bd. 2, 28: losen und lüsnen, Sappada lisnen zu ahd. hlusinôn.

11 In den Mundartgedichten von L. Henziger, Im Oberlond douba, Innsbruck o.J., für Paznaun bzw. Landeck loosna 161, glousnat 139; auch im Stanzertal gilt lousna.

12 Vgl. RN 2, 454 Losi, auch bei W. Vogt, Vbger. Flurnamembuch I/2, Silbertal (Montafon) Nr. $380 \mathrm{f}$. etc.; ähnlich auch lus m. 'durch das Los bestimmter Anteil an Grund und Boden' sowie Luss f. 'Lauer (der Jäger)' nach Idiotikon 3, 1455.

13 Kurze Verba werden nicht selten auf diese Weise "verlängert“ wie etwa tir. mienen' 'mühen', Schatz 434, oder im Inntal dann dran(en), Schatz 132; vgl. auch J. Schatz, Die Mundart von Imst, Straßburg 1897, 25. Gegen einen durch Randlage bedingten Archaismus spricht der Tonvokal, der auf ahd. hlosên verweist. Auch K. Finsterwalder nimmt in diesem Gebiet "sprachliche Eigenschöpfungen" an, belegt mit parg 'Berg' etc., Tiroler Ortsnamenkunde I, Innsbruck 1990, 12.

14 Unhaltbar sind allerdings die Schlußfolgerungen, die E. Gamillscheg aus einigen wenigen Vintschgauer Namen ableiten will, vgl. Die romanischen Namen des Untervintschgaus, in: Festschrift zum 19. Neuphilologentag, Berlin 1924, 34-59. Die Bedeutung des Verkehrs im Mittelalter erhellt der wichtige Aufsatz von O. Clavadetscher, Flurnamen als Zeugen ehemaligen Königsgutes in Rätien, in: Die Alpen in der europäischen Geschichte des Mittelalters, Konstanz-Stuttgart 1965, 141-158 (= Vorträge und Forschungen, 10). Zwischen der Übernahme von rtr. Dorfnamen ins Deutsche und dem Wechsel der Umgangssprache liegt meist eine lange Periode der Zweisprachigkeit, wie die Urkunden oder auch der Sprachgebrauch im Engadin belegen. 
Verba, die nicht aus dem Deutschen kommen können und die im Engadin sehr ähnlich klingen. Ein priasch $\alpha$ 'Brüllen (bes. der hungrigen Kühe)' kommt sicher von bargir und nicht aus einem nördlichen mnd. bresch 'Krach, Gebrüll' (Jutz 1, 450), wie die enge, sehr spezifische Bedeutung und der Lautstand ziemlich sicher beweisen. Das Reliktwort, im Rätoromanischen mit erweitertem Präsens (inchoatives -ISC- in der 1.-3. Pers. Sing. und 3. Pers. Plur., also bargèscha nach Lutta 186), wurde beim Sprachwechsel offenbar in der Lautgestalt dieser häufigen Personalformen übernommen und nicht etwa in der des Infinitivs. Es ist von *BRAGIRE (REW 1261) auszugehen, das leider im DRG erst unter sbragir behandelt werden wird, und REW 1038 brietsche ist zu streichen ${ }^{15}$. Das Relikt beweist Kontinuität und Kontakte in der mündlichen Sprachform gerade im Bereich und Gebrauch von verbalen Dialogformen, die auch bei anderen semantisch vergleichbaren Verba zu beobachten sind.

Ein gräzen 'zorniges Weinen, Schreien der Kinder, auch der Schweine beim Schlachten' (Jutz 1, 1235; Idiotikon 2, 835) hat dagegen als Erbwort einen viel weiteren Geltungsbereich, wie auch Fischer $(4,663 \mathrm{zu}$ krächzen) und Kluge-Seebold (408) nahelegen. Das erweist sein ungleich größerer Bedeutungsumfang und die weniger spezifische Bedeutung ebenso wie die geographische Verbreitung, denn gräzen gilt auch im Rheintal, am Bodensee etc. Nicht so klar liegt der Sachverhalt bei anderen Verba für 'weinen' wie etwa vbg. rära, plära, briegga, bella. Wenn Verba für eine "hörbare" Tätigkeit besonders leicht onomatopoetisch abgelenkt werden, dann müßte das auch für alemannische Verba gelten.

Ein anderes Verb, das aus einem ähnlichen semantischen Bereich kommt, ist schwerer zu beurteilen: ronschen (Jutz 2,754) 'unruhig auf seinem Sitz herumwetzen; sich im Bett wälzen' (Frastanz, Bludenz, Gisingen); 'sich müßig herumtreiben' (Thal-Sulzberg). Diese Angaben sind ungenau und ergeben kein klares Bild; sie zeigen überdies, daß etymologische Vorstellungen auch die Wortbeschreibung beeinträchtigen können, ohne daß es dem Lexikologen überhaupt bewußt geworden sein dürfte.

Das Zeitwort ronsche ist mir von meinem Heimatdialekt her geläufig, wird im Walgau [ronž $\alpha$ ] gesprochen und meint primär 'Geräusch und die Bewegung der Kühe, die sich am Barn, an einem Pfosten etc. reiben', fig. dann auch 'Herumwetzen der Kinder', wie Jutz 2, 754 anführt. Er ging jedoch von der marginalen, schon abgeleiteten und abgelenkten Bedeutung 'sich müßig herumtreiben' aus, die nur im Unterland (als rontschen) zu belegen ist und die vom hereinwirkenden dt. ranzen kommen dürfte (Jutz 2, 645 f.;Kluge-Seebold 581). Im Walgau wird das Verb nicht mit stimmloser Affrikate, sondern mit dem stimmhaften Reibelaut -ž- gesprochen, einem romanischen Reliktphonem, und es hat eine andere, sehr enge Bedeutung: Es geht daher primär um ein anderes Wort und um die im Sprachkontakt häufige Konvergenz. Die Bezugssphäre (Stall, Kinderstube),

15 Vgl. DRG 2, 178 bargentar und DRG 2, 314 berglir, das auch hier semantisch hereinspielen dürfte. 
die Lautform und die Verbreitung sprechen ganz eindeutig für ein rätoromanisches Reliktwort. Am ehesten ist von engad. ruojer 'beißen, nagen' auszugehen, das in Oberitalien wie auch im Rätoromanischen mehrfach vertreten ist (vgl. REW 7358 RODERE und $7380 *$ ROSICARE). Das $-n$-ist entweder aus dem falsch redressierten Langvokal oder über Einfluß von ruogna 'Räude, Krätze' (Pallioppi 626) zu erklären, das im Unterengadin als altes rätoromanisches Lehnwort gruscha 'Krätze' < langob. *hrudia hereinwirken könnte (DRG 7, 885 ff. und RN 2, 173). J. Jud (VR 7, 88) nennt dazu umerusche 'umherkriechen, -rutschen' (Obersaxen) aus dem Walser Adstrat. Auch (Ofa)rüsche n. 'Ofenkrücke', nach Jutz 2, $794 \mathrm{f}$. und C. Mätzler ${ }^{16}$ sicher ein Reliktwort, wie engad. ruschen 'Schüreisen' (Peer 404) zeigt, gehört lautlich wie auch begrifflich in diesen Bereich ${ }^{17}$.

Das tir. rúeschn 'raschelnd herumsuchen; übereilt arbeiten', dazu riesch(e)n (Pustertal, Etsch, Defreggen nach Schatz 495) 'den Backofen auskratzen, reinigen' und das Übertragen auf lebhafte Kinder stimmt semantisch recht gut zu furl. roseá 'suchen'. Den Übergang bildet rudié oder ruzié 'rodere, rosicchiare' im Comelico ${ }^{18}$, das auch den stimmhaften Reibelaut recht plausibel macht, der im östlichen südbairischen Tirol dann stimmlos werden muß.

Ein weiteres Verbum, das über heutige Landesgrenzen hinausreicht, aber meist mit dem Hinweis auf seine lautmalende Form nicht näher auf seinen Ursprung und die weiteren Zusammenhänge untersucht wird, ist $p f \hat{u} f a$ 'fauchen'. Es gibt in Vorarlberg neben sehr ähnlich klingendem pfûchen ${ }^{19}$, das von Jutz 1, 344 für das ganze Unterland und den Vorderwald belegt wird als 'fauchen, zischen (von Katzen, Gänsen), Geräusch des entweichenden Dampfes, des heißen Eisens beim Abschrecken', auch die Variante $p f u f a$ (Jutz 1, 345). Diese hat eine merklich verschiedene Bedeutungsstruktur von der erstgenannten Form und meint zuerst 'zornig aufbrausen (von Menschen)', bes. an-, dann 'keuchen, schwer atmen (von beleibten Personen etc.)'. Für Lustenau wird auch pfumpfe angegeben, das Interferenzen und Spielformen im Randgebiet unter dem Kummenberg verdeutlicht.

In Tirol finde ich pfûfe 'fauchen, blasen' (Paznaun, Stanzertal), das auch dem Oberen Gericht nicht fremd sein kann wegen Pfûferle n. 'leicht hingewehter Schnee' (Prutz), beides bei Schatz 75. Daneben gibt es mehrere mit fauchen verbundene Intensivformen wie pfüchezn (Tux) 'zischend aufbrausen', pfüehezn (Tux) 'sich schnaubend vom Gestank abwenden' (Schatz 75) mit dem für Tirol bezeichnenden Ergebnis von germ. -atjan > bair. -ezn (Henzen 1965, $226 \mathrm{ff}$.).

16 Romanisches Wortgut in den Mundarten Vorarlbergs, Innsbruck 1968, 41. Weiterführend ist E. Gabriel, Die alemannisch-bairische Sprachgrenze am Arlberg, in: Alem. Jahrbuch 1971/72, 239-60, wo insbesondere vorrömische Relikte behandelt werden.

17 Das Verbum hat sicher nichts zu tun mit gall. *rusca 'Baumrinde', REW 7456, wie H. Klausmann und T. Krefeld in Raetia antiqua et moderna, Fs. für Th. W. Elwert, Tübingen 1986, 127 vermuten, ebenso wenig mit rtr. rusna 'Loch', vgl. RN 2, 286. Der Aufsatz gibt Wortkarten aus unserm Gebiet.

18 Vgl. E. de Lorenzo Tobolo, Dizionario del dialetto ladino di Comèlico Superiore, Bologna 1977. Auch W. Th. Elwert, Die Mundart des Fassa-Tals, Wiesbaden ${ }^{2} 1972,220$ als ruser 'kratzen'.

$19 \mathrm{Zu}$ den deutschen Iterativbildungen etwa W. Henzen, Deutsche Wortbildung, Tübingen ${ }^{3} 1965$, Anlautverstärkung bes. 213. Man sagt auch umgangssprachlich gleich pfupfauf sein für 'leicht zornig werden'. 
In Südvorarlberg muß man $p f \hat{u} f a$ 'keuchen', auch 'fauchen (von Tieren, dgl. fig.)' von $p f \hat{u} c h a$ mit der Intensivform $p f \hat{u} f z g a$ 'fauchen', fig. 'jdn. anfahren' trennen ${ }^{20}$. Die Wortbildung allein vermag nicht zu erklären, warum in der einfachen Grundform ch durch $f$ ersetzt wurde, denn die Konsonantenfolge ist sicher sekundär und auch expressiv bedingt. Es gibt aber auch noch den Aspekt der Umsetzung in ein anderes Phonemsystem, der bei Hereinwirken eines romanischen Reliktwortes wie búfa in unserem Dreiländereck nicht übersehen werden darf.

Im Rätoromanischen des Engadins gibt es offenbar boffer 'stark Atem holen, keuchen; blasen' nach Pallioppi 110, das wie ital. buffare ursprünglich mit dem expressiven, bezeichnenden $b-f$ gebildet sein dürfte ${ }^{21}$. Wenn ein deutschromanischer Zusammenhang besteht, was zu vermuten ist, dann ist der romanische Anlaut $b$ - unter lautmalendem (bairischem ?) Einfluß zu $b f-, p f$-geworden; dies entspricht aber nicht der regelrechten Umsetzung. Eine gewisse Zweigleisigkeit kann hier in der Entsprechung von $f$ - liegen, das in unserem Gebiet häufig als $p f$ - aufscheint wie in vbg. Pfö $(n) \mathrm{m}$ (Jutz I, 962 f.) <FAVONIUS, tir. Pfiem u. ä. 'Föhn' (Schatz 69) oder vbg. Pfurre 22 n. < FURIA (Jutz 1, 346 f.; vgl. pfurrn Schatz 76) u.a. gegenüber $p$-, verschoben als $p f$ - in sehr frühen Übernahmen und Entlehnungen im Süddeutschen wie etwa Pfulfa 'Polster' (Montafon, Jutz 1, 345). Für ein Relikt spricht in diesem Fall meines Erachtens vor allem die enge Sonderbedeutung 'keuchen, schwer atmen', die rtr. buffar mit $p f u f a$ verbindet $^{23}$. Eine Sonderbedeutung dieser Art bildet sich in einem so kleinen Gebiet mit den erwiesenen engeren Kontakten auch in früherer Zeit nicht mehrfach und unabhängig heraus. Spätere lautliche und semantische Ablenkung in Verbindung mit neuer Wortmotivation (Konvergenz) ist bei Relikten so häufig anzutreffen, daß es dazu wohl nicht breiterer Ausführungen bedarf.

Ähnlich scheinen die Dinge bei einem weiteren Verb menschlicher Lautgebung zu liegen, nämlich bei vbg. beffen, das viel eher von rom. beff- (REW 1017) kommt als von dt. betzgen, wie Jutz I, $286 \mathrm{f}$. vorschlägt. Das Wort lebt in Vorarlberg als peffl $\alpha$ (Montafon), geläufiger aber als peftsg $\alpha$ und meint:

1.) 'viel (und bes. keifend, scheltend) reden' (Schruns), 'kleinlich, nörgelnd zanken' (Gaißau);

2.) 'kläffen, andauernd bellen (bes. von kleinen Hunden)'; dazu gibt es die Ableitungen Péffle, Béftske(r) als Nomen agentis und Pefflete f. (Montafon) als Nomen actionis.

20 Es gibt in Vorarlberg neben fuftsk 'fünfzig' auch die seltenere Variante fuchtsk, Jutz 1, 1023; analoge Konsonantenverbindungen in Verba wie schmatzga 'schmatzen' $<$ mhd. smackezen (Jutz 2, 980) oder gatzga 'gackern' (Jutz 1, 1068) weisen eindeutig Metathese auf, die kleinräumig charakteristisch zu sein scheint, ebenso gritschga 'knirschen' (Jutz 1, 1242; grutschga bei Schatz 251) u.a.

21 Vgl. G. Devoto, Avviamento alla etimologia italiana, Firenze 1968.

22 Vgl. Verf. in: Mundart und Name im Sprachkontakt, Fs. für M. Hornung, Wien 1990, 118.

23 Eine ähnliche Bedeutung hat vbg. pisten 'schwer und tief, geräuschvoll atmen, ächzen, stöhnen' (Jutz 1, 365), das aber den Anlaut nicht veränderte. Es reicht auch ins Paznaun hinüber, Schatz 82, aber im Oberinntal gilt dafür kreisten, Schatz 356. 
In Tirol ist pefern 'nachmaulen, eine Rede spottend und rasch nachäffen' (Inntal, Schatz 54) zu belegen, pefn (Ötztal) und pefe (oberes Oberinntal) 'brummen, schelten'; pefin 'bellen, geifern, nachmaulen' (Unterinntal) und dazu pefla m. 'Kläffer'. In Tannheim gilt béfze, im oberen Lechtal péffzga wie in Vorarlberg. Auch das pustert. pefl (Kiens) 'zannen, Gesichter schneiden' gehört wohl dazu, aber nicht pelfern, bellen u.ä. Die ältere Bedeutung in Westtirol und im Südalemannischen ist wohl 'schelten', auch im Inntal, wo dann 'nachäffen' und 'bellen' dazukommt. Verbreitung und Semantik legen eher ein Lehnwort als ein Reliktwort nahe. Als Grundform scheint beffeggiare vorzuliegen.

Das engad. beffer, $s$ - 'spotten, ver-' und beffager 'spotten' aus beffa + IDIARE (DRG 2, $283 \mathrm{f}$.) sind vom Substantiv beffa 'Hohn, Spott' abgeleitet. Schon Oswald von Wolkenstein (+1445) kennt "centza befiw - an allen spot", übersetzt es aber, da es damals eher noch Fremdwort als Lehnwort war, jedoch dann ins Südbairische übernommen worden sein muß. Die Verba sind schon wegen der Varianten dt. befe(r)n und -ez(g)en sekundär, haben eine andere Valenz als das rom. beffar und führen auch semantisch 'Schelte' weiter, nicht das spätere 'Spott'.

Wenn Romanisten und Germanisten gerade bei der Interpretation von Relikten, Entlehnungen und den folgenden, vielfach konvergierenden Interferenzen nicht selten zu entgegengesetzten Resultaten kommen, so liegt dem nicht unbedingt die böse Absicht zugrunde, ein Wort auf Biegen und Brechen der vertretenen Sprache einzuverleiben. Meist haben solche Wörter ja doch mehr oder weniger Heimatrecht in mehreren Kontaktsprachen, wie der unbefangene Sprecher des lokalen Sprachstandes ja auch zeigt in seinen Aneignungsversuchen, die wir Volks- oder Paraetymologien nennen. Häufig liegt die Ursache lediglich in den Assoziationen, die das Wort beim Bearbeiter auslöst. Wir suchen wegen unserer jeweiligen engen einzelphilologischen Ausrichtung (man könnte auch sagen: déformation professionnelle oder Einäugigkeit) nach Auswegen, die sich vielleicht aus anderem, breiterem Blickwinkel als Sackgassen erweisen. Man spielt mit Wortkreuzungen, mit Laut- und Semkombinationen, und oft, allzu oft werden lautmalende Schallwörter strapaziert, bis jemand - oft zufällig - den überzeugenden Ansatz findet. Ein Eingehen auf Kontaktsprachen in alten Überlagerungsarealen verspricht jedenfalls bessere Ergebnisse, wie uns nicht zuletzt mancher Kollege aus dem so vielfach überschichteten Balkan gezeigt hat.

\author{
Povzetek
}

\title{
ROMANSKE JEZIKOVNE OSTALINE V TRIKOTU MED AVSTRIJO, ŠVICO IN ITALIJO
}

Avtor raziskuje leksikalne ostaline $v$ danes nemško govorečih visokih alpskih dolinah okrog tromeje Avstrija-Italija-Svica (Montafon, Arlberg, dolina zgornjega Inna, dolina gornje Adiže). Ostaline nekdanjega romanskega govora so vidne že v toponimih, npr. Montafon MONS + TUBUS ali Paznáun, kjer ugotavljamo lat. leksem PUTEUS, zaznavne pa so romanske prvine tudi v občnih imenih, npr. $v$ izrazu za topli veter $P$ fön iz lat. FAVONIUS (VENTUS). Avtor opozarja tudi na paraetimološke razlage: čprav so lahko zmotne, velikokrat odkrivajo (zaradi asociacij) psihološke vzgone v jeziku. 
$\stackrel{\circ}{\circ}$

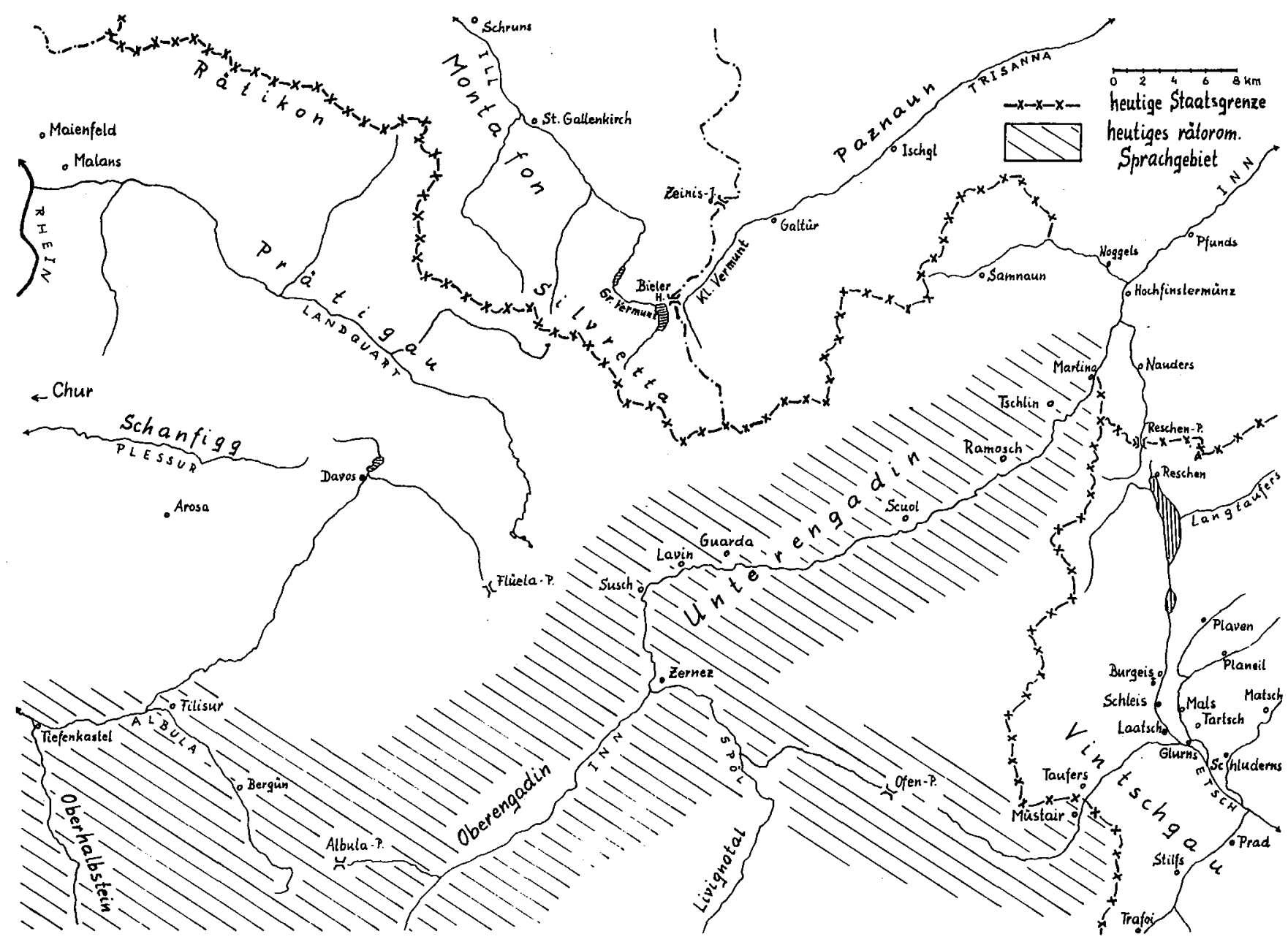

